\title{
COMMUNICATION
}

\section{Lésion radioclaire mandibulaire : à propos d'un cas atypique de tumeur odontogène.}

\author{
De Chargères B1', Genevière H, Lescaille G, Descroix V
}

1. Service d'Odontologie, Groupe Hospitalier Pitié-Salpêtrière, APHP - Université Pierre et Marie Curie UPMC - Paris

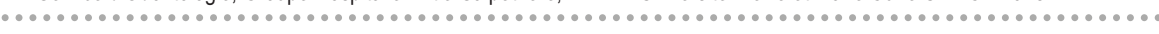

\section{Introduction}

Si les lésions radioclaires peri-apicales sont fréquemment d'origine endodontique, certaines tumeurs osseuses peuvent avoir des aspects radiologiques proches et nécessitent un diagnostic approprié.

\section{Observation}

Cette étude rapporte le cas d'une lésion péri-apicale de découverte fortuite à l'orthopantomogramme chez une patiente de 19 ans après deux ans de suivi en orthodontie. Le département d'endodontie ayant écarté le diagnostic de lésion inflammatoire péri-apicale d'origine endodontique sur 32, une imagerie $3 D$ de type $C B C T$ était réalisée. L'imagerie révélait une lésion intra-osseuse de tonalité mixte (radio-claire avec quelques inclusions radio-opaques), sans délimitation nette, associée à une résorption de la racine dentaire de 32. Les diagnostics différentiels évoqués étaient alors principalement : le fibrome ossifiant (F0), la dysplasie fibreuse (DF), la dysplasie osseuse (DO). Cependant, compte tenu de l'image radiologique et du caractère rapidement évolutif (absence de lésion deux ans auparavant), une biopsie osseuse a tout d'abord été réalisée afin d'écarter un processus malin. Le résultat anatomopathologique était en faveur d'une lésion fibro-osseuse de type FO. L'exerèse de la lésion sous anesthésie locale a été décidée après traitement endodontique de la 32. Lors de la chirurgie, un lambeau de pleine épaisseur était levé de 34 à 42, permettant d'accéder à une lésion mal délimitée sans réel plan de clivage. Après élimination d'un tissu fibreux et calcifié par endroit, un curetage par fraisage des parois ainsi qu'une résection radiculaire de 32 ont été réalisés. Les suites opératoires ont été simples et nous avons réalisé des examens radiologiques de contrôle. Compte tenu d'une cicatrisation partielle à un an, la patiente sera revue dans 3 mois avec de nouveaux examens radiologiques afin d'affirmer ou non le diagnostic de récidive locale.

\section{Discussion}

Le FO est une tumeur fibro-osseuse odontogène des maxillaires en lien avec une origine possible dans une anomalie de développement du ligament desmodontal. II se caractérise par un mode d'évolution souvent lent et asymptomatique et son diagnostic repose à la fois sur des critères épidémiologiques, cliniques, radiologiques et histologique. Cette lésion prédomine chez la femme, notamment dans une localisation prémolo-molaire mandibulaire et se présente le pus souvent par une masse calcifiée bien délimitée, sans récidive après chirurgie. Dans notre cas, bien que les analyses histologiques soient en faveur d'une lésion de FO, le diagnostic différentiel avec une lésion de dysplasie fibreuse est également à discuter compte tenu de l'absence de délimitation macroscopique de la lésion avec les tissus environnants. Afin d'éliminer une forme polystotique, nous avons confirmé que la patiente ne présentait pas les autres critères classiques du syndrome de McCune-Albright (absence de taches cutanées « café au lait » et pseudo puberté précoce).

\section{Mots clés}

tumeurs osseuses odontogènes, fibrome ossifiant, dysplasie fibreuse, dysplasie cémento-osseuses.

bdechargeres@gmail.com

Bibliographie

- J Guillet et al. Med Buccale Chir Buccale 2010 ;20:129-134

- B Courtois et al. Med Buccale Chir Buccale 2004; 10: 21-30

- S Haitami et al. Med Buccale Chir Buccale 2015;21:183-187

(C) The authors, published by EDP Sciences. This is an Open Access article distributed under the terms of the Creative Commons Attribution License 4.0 (http://creativecommons.org/licenses/by/4.0/). 